\title{
Association between Obesity, Surgical Route, and Perioperative Outcomes in Patients with Uterine Cancer
}

\author{
Entidhar Al Sawah, ${ }^{1}$ Jason L. Salemi, ${ }^{2}$ Mitchel Hoffman, ${ }^{3}$ \\ Anthony N. Imudia, ${ }^{1}$ and Emad Mikhail (iD) ${ }^{1}$ \\ ${ }^{1}$ Department of Obstetrics and Gynecology, University of South Florida Morsani College of Medicine, Tampa, FL, USA \\ ${ }^{2}$ Department of Family and Community Medicine, Baylor College of Medicine, Houston, TX, USA \\ ${ }^{3}$ Division of Gynecologic Oncology, Moffitt Cancer Center, University of South Florida, Tampa, FL, USA
}

Correspondence should be addressed to Emad Mikhail; emikhail@health.usf.edu

Received 17 January 2018; Revised 14 April 2018; Accepted 10 May 2018; Published 19 June 2018

Academic Editor: Saad Amer

Copyright (C) 2018 Entidhar Al Sawah et al. This is an open access article distributed under the Creative Commons Attribution License, which permits unrestricted use, distribution, and reproduction in any medium, provided the original work is properly cited.

\begin{abstract}
Objective. To study temporal trends of hysterectomy routes performed for uterine cancer and their associations with body mass index (BMI) and perioperative morbidity. Methods. A retrospective review of the American College of Surgeons-National Surgical Quality Improvement Program (ACS-NSQIP) 2005-2013 databases was conducted. All patients who were 18 years old and older with a diagnosis of uterine cancer and underwent hysterectomy were identified using ICD-9-CM and CPT codes. Surgical route was classified into four groups: total abdominal hysterectomy (TAH), total vaginal hysterectomy (TVH), laparoscopic assisted vaginal hysterectomy (LAVH), and total laparoscopic hysterectomy (TLH) including both conventional and robotically assisted. Patients were then stratified according to BMI. Results. 7199 records were included in the study. TLH was the most commonly performed route of hysterectomy regardless of BMI, with proportions of $50.9 \%, 48.9 \%, 50.4 \%$, and $51.2 \%$ in ideal, overweight, obese, and morbidly obese patients, respectively. The median operative time for TAH was 2.2 hours compared to 2.7 hours for TLH ( $p<0.01$ ). The median length of stay for TAH was 3 days compared to 1 day for TLH $(p<0.01)$. The percentage of patients with an adverse outcome (composite indicator including transfusion, deep venous thrombosis, and infection) was 17.1 versus 3.7 for TAH and TLH, respectively $(p<0.01)$. Conclusion. During the last decade, TLH has been increasingly performed in women with uterine cancer. The increased adoption of TLH was seen in all BMI subgroups.
\end{abstract}

\section{Introduction}

Uterine cancer is the most common gynecologic cancer in USA [1], with the median age at presentation being 60 years [2]. Depending on the stage and grade, surgery is the mainstay of treatment of uterine tumors, with or without subsequent radiation. Early stage uterine cancer can be managed safely with conventional as well as robotically assisted laparoscopic approaches $[3,4]$.

Despite the evidence-based benefits associated with minimally invasive gynecologic surgical approaches, laparotomy remains the route of choice in more than $60 \%$ of the 600,000 hysterectomy procedures performed annually in USA [5]. The rate of abdominal hysterectomy in USA between 2003 and 2005 was still over $60 \%$ and only $12-14 \%$ of hysterectomies were being performed laparoscopically [6].

Greater degree of obesity is a well-known risk factor for the development of uterine cancer $[7,8]$. Surgery for uterine cancer in obese patients can pose significant intra- and postoperative challenges to the surgeon. Obesity is associated with a higher rate of conversion of laparoscopic surgery to laparotomy and a lower completion rate of lymph node dissection [9].

Although obesity is associated with higher incidence of uterine cancer and recent studies have reported that the rate of abdominal hysterectomy performed for benign indications is increased in obese patients [10], the extent to which obesity plays a role in the surgical management of 
- STUDY POPULATION -

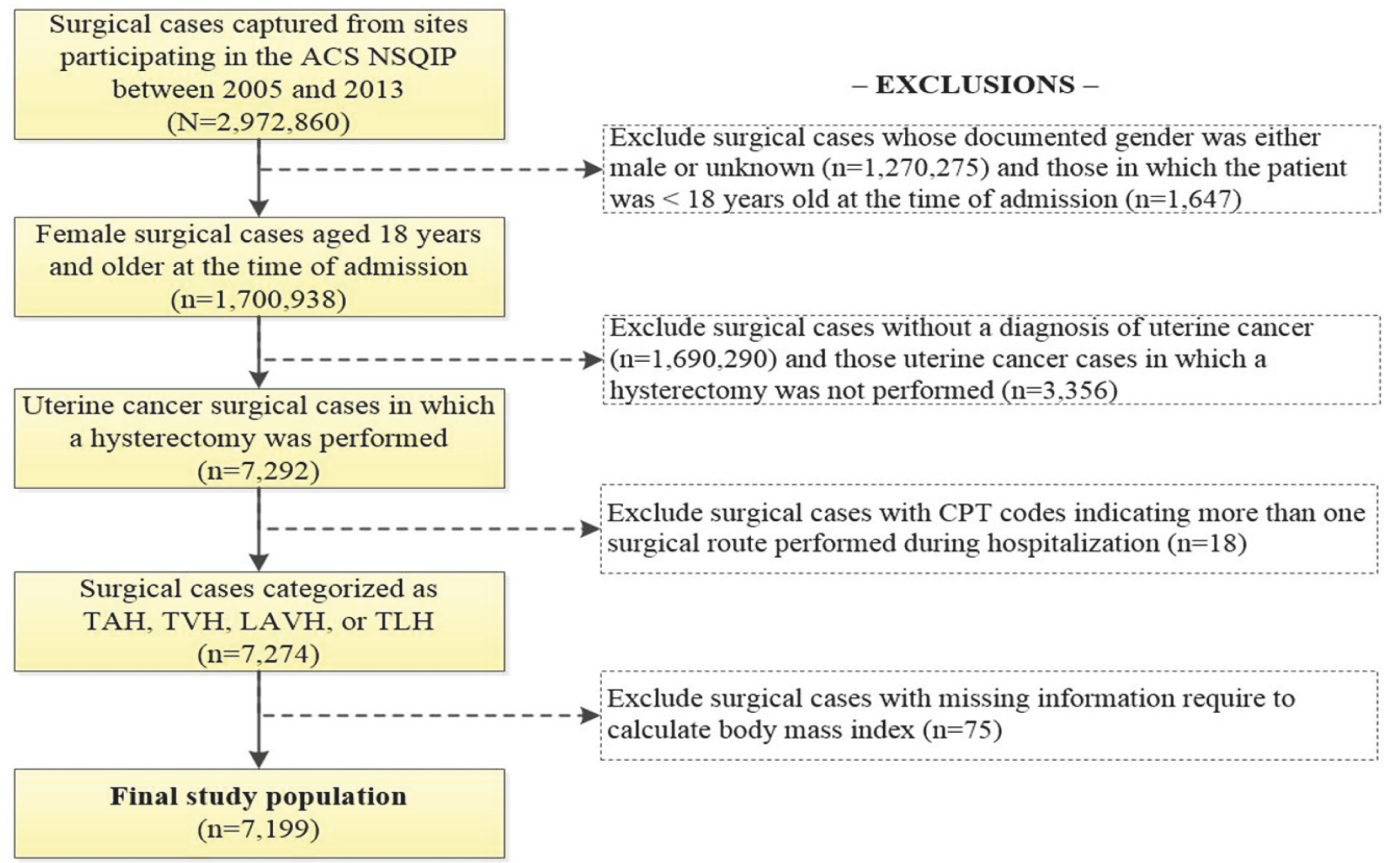

FIGURE 1: Flow diagram representing the final determination of all patients in which a hysterectomy was performed among patients with a diagnosis of uterine cancer, ACS-NSQIP, 2005-2013. ACS-NSQIP: American College of Surgeons-National Surgical Quality Improvement Program; TAH: total abdominal hysterectomy; TVH: total vaginal hysterectomy; LAVH: laparoscopic assisted vaginal hysterectomy; TLH: total laparoscopic hysterectomy.

uterine cancer is currently unknown. We hypothesize that, with increased obesity, the proportion of minimally invasive surgical approach might decrease. Therefore, in this study, we explore the association between obesity and surgical route for the treatment of uterine cancer, and we describe the extent to which the rate of perioperative complications differs by obesity status.

\section{Materials and Methods}

After obtaining exempt status from the University of South Florida's institutional review board, we used the American College of Surgeons-National Surgical Quality Improvement Program (ACS NSQIP) database from 2005 to 2013 to conduct a retrospective, cross-sectional analysis of female patients with uterine cancer who underwent hysterectomy. ACS-NSQIP is a publically available and deidentified database created as part of a quality improvement initiative originally developed by the Veterans' Health Administration in 1991 and adopted by the American College of Surgeons in $2001[11,12]$. The database includes more than 450 participating community and academic hospitals nationwide. Data captured include but are not limited to demographics, comorbidities, laboratory values, and operative variables, as well as 30-day postoperative outcomes, complications, mortality, reoperation, and length of stay. Quality improvement and assurance protocols include routine auditing and the use of specially trained surgical nurses to record patient variables.
A random 8-day sampling method is used to ensure that a diverse range of surgical procedures is captured.

In patients aged 18 years or above, we identified women with uterine cancer using the principal postoperative diagnosis (ICD-9-CM code 182.0, 182.1, or 182.8). We then used current procedural terminology (CPT) procedure codes to identify 7,292 surgical cases in which hysterectomy was performed and then specific codes were used to subclassify cases as (1) total abdominal hysterectomy (TAH, 58150 and 58200); (2) total vaginal hysterectomy (TVH, 58260, 58262, 58263, 58270, 58275, 58280, 58290, 58291, 58292, and 58294); (3) laparoscopic assisted vaginal hysterectomy (LAVH, 58550, 58552, 58553, and 58554); and (4) total laparoscopic hysterectomy (TLH, 58570, 58571, 58572, and 58573). We excluded 18 cases $(0.2 \%)$ with CPT codes indicating more than one surgical route performed. Due to absence of specific CPT codes, we could not differentiate between robotically assisted laparoscopic hysterectomies from TLH; therefore, these groups are combined into a single group (TLH) (Figure 1).

Body mass index (BMI) was calculated as [(weight in pounds $\left.) /(\text { height in inches })^{2}\right) \times 703$ ], and then patients were classified according to BMI as follows: normal and underweight $(<25)$, overweight (25-29.9), obese (classes I and II; 30-39.9), and morbid obesity (class III; $\geq 40$ ). We excluded 75 cases (1.0\%) for which BMI could not be calculated due to missing information on presurgical weight and height. Data analyzed included patient age, race/ethnicity, operative time, length of hospital stay, blood transfusion, 


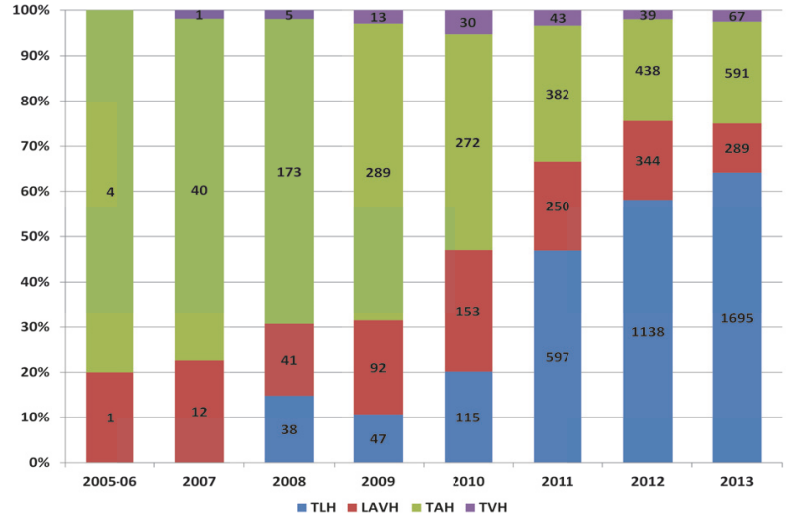

FIGURE 2: Frequency and proportion of different types of hysterectomies performed among patients with a diagnosis of uterine cancer, ACS-NSQIP, 2005-2013. TAH: total abdominal hysterectomy; TVH: total vaginal hysterectomy; LAVH: laparoscopic assisted vaginal hysterectomy; TLH: total laparoscopic hysterectomy.

development of deep venous thrombosis, and development of surgical infection. Infection types included superficial surgical site (involving only skin or subcutaneous tissue) and deep incisional surgical site (involving deep soft tissues). When data were available (2011 or later), we also captured 30day readmissions.

Descriptive statistics were used to describe the frequency and temporal trends in surgical approaches in the entire study population and stratified by patient's BMI level. Differences in the distribution of selected patient sociodemographic and hospital characteristics and in the rates of clinical outcomes by surgical approach were assessed using either a WilcoxonMann-Whitney test (continuous variables) or chi-square test (categorical variables). For each hysterectomy route, we compared the rate of perioperative outcomes across levels of patient's BMI. All statistical analyses were conducted using SAS version 9.4 (SAS Institute, Inc., Cary, NC), using a 5\% type I error rate and two-sided hypothesis tests. STrengthening the Reporting of OBservational studies in Epidemiology (STROBE) guidelines for reporting observational studies were followed for this study [13].

\section{Results}

Between 2005 and 2013, we identified a total of 7,199 uterine cancer surgical cases managed with hysterectomy and with documented presurgical weight and height (Figure 1). The most common route of hysterectomy was TLH (50.4\%), followed by TAH (30.4\%), LAVH (16.4\%), and TVH (2.8\%). During the study period, we observed a relative increase in the use of the TLH route (from $15 \%$ in 2008 to $64 \%$ in 2013) and a concomitant relative decrease in the use of TAH (from $67 \%$ in 2008 to $22 \%$ in 2013) (Figure 2). Until 2013, the proportion of hysterectomies using the LAVH route was relatively constant over time, as was the small proportion of surgical cases in which TVH was performed.

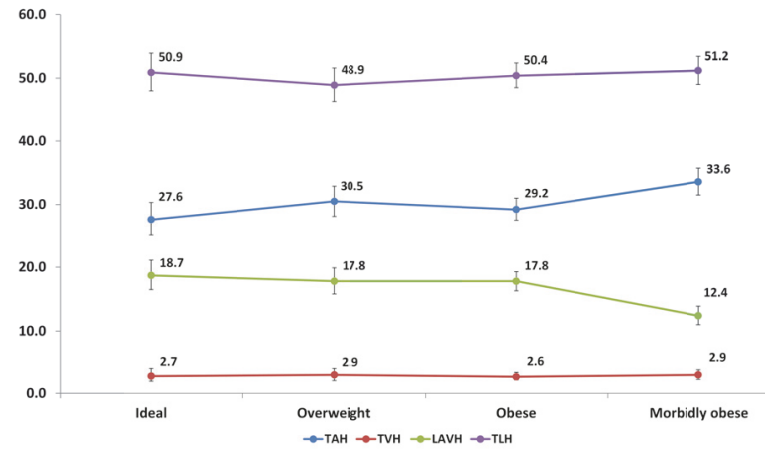

FIGURE 3: Rates of different types of hysterectomy performed among patients with a diagnosis of uterine cancer, by patient's body mass index, ACS-NSQIP, 2005-2013.

Total laparoscopic hysterectomy was the most performed procedure regardless of BMI, occurring in 50.9\%, 48.9\%, $50.4 \%$, and $51.2 \%$ of patients with ideal, overweight, obese, and morbidly obese BMI, respectively (Figure 3 ). The overall rate of TAH was $30 \%$; however, the rate tended to be slightly higher in patients who were morbidly obese $(33.6 \%)$ relative to other patients. This increase in the rate of TAH in morbidly obese patients was at the expense of LAVH (12.4\%); the LAVH rate among the morbidly obese was statistically significantly lower than the LAVH rate in each of the other BMI categories.

The median patient BMI differed significantly by surgical approach; patients who underwent TAH had overall higher BMI $\left(34.0 \mathrm{~kg} / \mathrm{m}^{2}\right)$ compared to those undergoing TVH (33.1 $\left.\mathrm{kg} / \mathrm{m}^{2}\right)$, LAVH $\left(32.2 \mathrm{~kg} / \mathrm{m}^{2}\right)$, and TLH $\left(33.7 \mathrm{~kg} / \mathrm{m}^{2}\right)$ (Table 1$)$. Patients undergoing TAH and LAVH were more likely to be nonwhite than patients undergoing TVH and TLH $(p<0.01)$. The median operative room time was statistically significantly shorter for TAH (2.2 hours) compared to TLH (2.7 hours). However, the median length of hospital stay for TAH was three times longer than TLH, TVH, or LAVH $(p<0.01)$. Over $15 \%$ of patients who underwent TAH stayed in the hospital for 6 days or longer, compared to only $1.3 \%$ of patients undergoing TLH $(p<0.01)$. The rates of several perioperative complications were increased significantly in patients who underwent TAH compared to TLH; these complications include transfusion $(10.3 \%$ versus $1.7 \%)(p<0.01)$, surgical site infection $(7.1 \%$ versus $1.7 \%)(p<0.01)$, and readmission within 30 days $(8.9 \%$ versus $3.8 \%)(p<0.01)$.

Regardless of the route of hysterectomy, patients who were morbidly obese, obese, or overweight tended to have statistically significantly longer operation times than patients who had an ideal BMI $(p<0.05)$. Similarly, postoperative infections, including superficial or deep surgical site infections, were more common in higher BMI categories when compared with ideal BMI $(p<0.05)$, and patients who were morbidly obese experienced substantially higher rates of any surgical site or wound infection (6.4\%) compared to patients who had an ideal BMI (1.6\%). The 30-day readmission rates were similar across all BMI categories (Table 2).

The rate of readmission and the rate of a composite infection outcome (including superficial surgical site infection, 


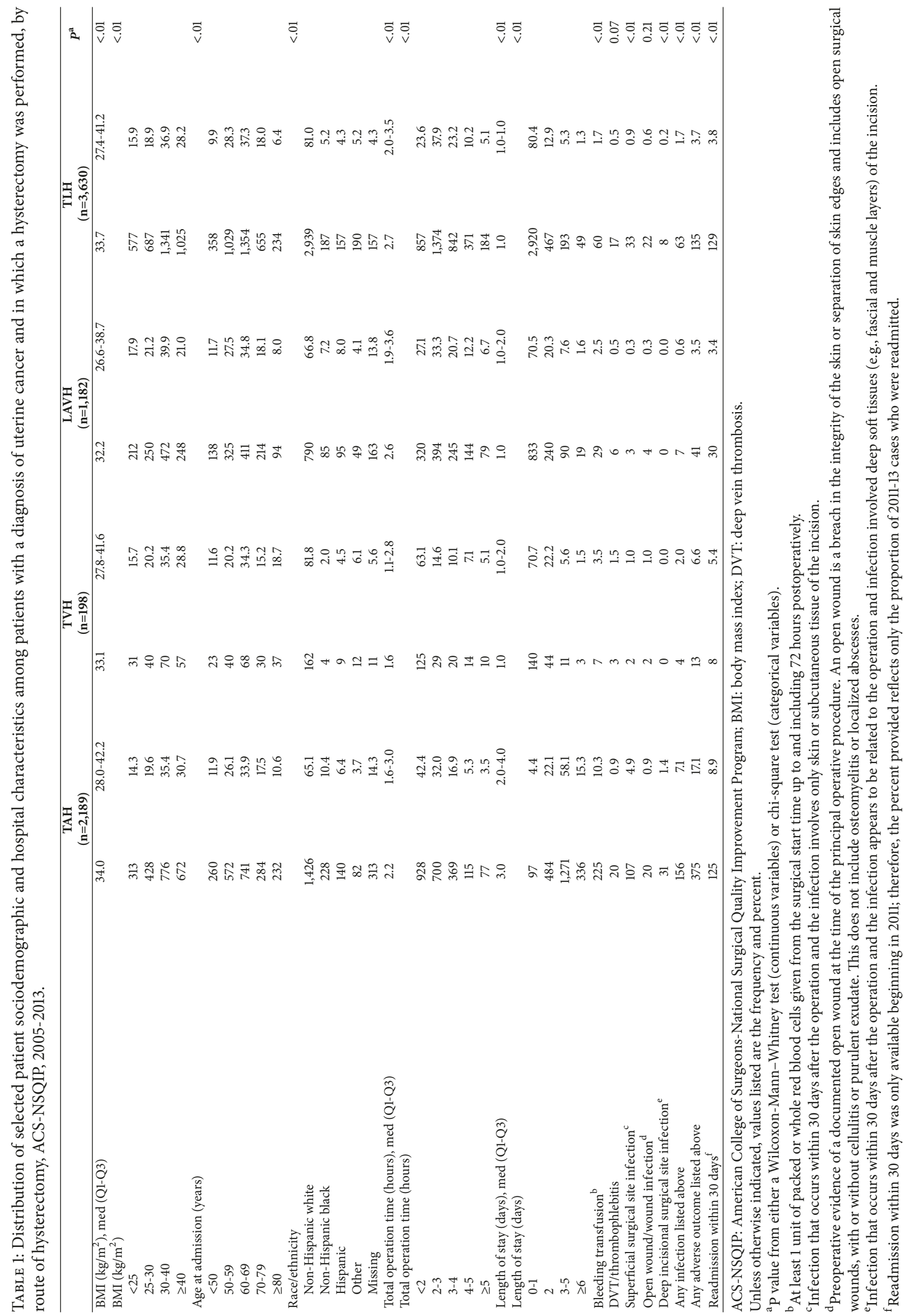


TABLE 2: Perioperative outcomes stratified by patient's body mass index, ACS-NSQIP, 2005-2013.

\begin{tabular}{|c|c|c|c|c|}
\hline \multirow[b]{2}{*}{ Characteristic/outcome } & \multicolumn{4}{|c|}{ BMI classification } \\
\hline & Ideal & Overweight & Obese & Morbidly obese \\
\hline Patient age (years) ${ }^{\mathrm{a}}$ & $63(55-74)$ & $65(57-73)$ & $63(57-71)$ & $60(54-65) *$ \\
\hline Operation time $(\mathrm{min})^{\mathrm{a}}$ & $137(100-189)$ & $143(102-193) *$ & $149(110-200) *$ & $164(124-214) *$ \\
\hline Length of hospital stay (days) ${ }^{\mathrm{a}}$ & $1(1-2)$ & $1(1-3)$ & $1(1-3)$ & $1(1-3) *$ \\
\hline Bleeding transf usion ${ }^{\mathrm{b}}$ & $79(7.0)$ & $70(5.0) *$ & $90(3.4) *$ & $82(4.1) *$ \\
\hline DVT/thrombophlebitis & $3(0.3)$ & $11(0.8)$ & $18(0.7)$ & $14(0.7)$ \\
\hline Superficial surgical site infection ${ }^{c}$ & $11(1.0)$ & $10(0.7)$ & $53(2.0) *$ & $71(3.5) *$ \\
\hline Open wound/wound infection ${ }^{\mathrm{d}}$ & $5(0.4)$ & $4(0.3)$ & $10(0.4)$ & $29(1.4) *$ \\
\hline Deep incisional surgical site infection $^{e}$ & $2(0.2)$ & $2(0.1)$ & $7(0.3)$ & $28(1.4) *$ \\
\hline Any infection listed above & $18(1.6)$ & $16(1.1)$ & $68(2.6)$ & $128(6.4) *$ \\
\hline Any adverse outcome listed above & $96(8.5)$ & $91(6.5)$ & $167(6.3) *$ & $210(10.5)$ \\
\hline Readmission within 30 days $^{\mathrm{f}}$ & $46(5.0)$ & $47(4.1)$ & $101(4.6)$ & $98(6.0)$ \\
\hline
\end{tabular}

ACS NSQIP = American College of Surgeons National Surgical Quality Improvement Program; BMI = body mass index; DVT = deep vein thrombosis . $* \mathrm{P}$-value $<0.05$ from either a Wilcoxon-Mann Whitney test (continuous variables) or chi-square test (categorical variables). For each outcome, three tests are performed: overweight vs. ideal BMI, obese vs. ideal BMI, morbidly obese vs. ideal BMI.

${ }^{\mathrm{a}}$ Values presented as median (Q1-Q3); all others are presented as frequency (\%).

${ }^{\mathrm{b}}$ At least 1 unit of packed or whole red blood cells given from the surgical start time up to and including 72 hours postoperatively.

${ }^{c}$ Infection that occurs within 30 days after the operation and the infection involves only skin or subcutaneous tissue of the incision.

${ }^{d}$ Preoperative evidence of a documented open wound at the time of the principal operative procedure. An open wound is a breach in the integrity of the skin or separation of skin edges and includes open surgical wounds, with or without cellulitis or purulent exudate. This does not include osteomyelitis or localized abscesses.

${ }^{\mathrm{e}}$ Infection that occurs within 30 days after the operation and the infection appears to be related to the operation and infection involved deep soft tissues (e.g., fascial and muscle layers) of the incision.

${ }^{\mathrm{f}}$ Readmission within 30 days was only available beginning in 2011; therefore, the percent provided reflects only the proportion of 2011-13 cases who were readmitted.

open wound/wound infection, and deep incisional surgical site infection) within 30 days were significantly lower for TLH and LAVH compared to TAH in all BMI subgroups except for the overweight group (Supplemental Tables S1-S4).

In comparing perioperative outcomes of TLH stratified by patient's BMI category, it was noted that increasing degree of obesity was associated with longer operative time. The mean operation time in patients of ideal weight $(145 \mathrm{~min})$ was shorter compared to overweight, obese, and morbidly obese women $(159,158$, and 171 minutes, resp., $p<0.05)$. Also, the rate of open surgical wound or wound infection was higher in the morbidly obese group $(1.3 \%)$ compared to ideal weight patients $(0.2 \%)(p<0.05)$. All other perioperative outcomes were not statistically significantly different across BMI levels (Table 3).

\section{Discussion}

In this study, using the ACS-NSQIP database, TLH (including conventional or robotically assisted) was found to be the most frequently chosen route for hysterectomy for surgical management of patients with uterine cancer. Performance of TLH increased from $16.5 \%$ in 2008 to $64.1 \%$ in 2013 . We also found that TLH is the most commonly chosen route regardless of the degree of obesity. Despite increased operative time compared to abdominal hysterectomy, the minimally invasive approach provided better perioperative outcomes manifesting in decreased length of hospital stay and decreased rates of transfusion, surgical site infection, and readmission within 30 days. The utilization of TLH was not negatively impacted by the degree of obesity, despite the increase in operative time and surgical infection.

The results from current study are consistent with data from the SEER database, which showed that performance of minimally invasive hysterectomy has increased from $9.3 \%$ in 2006 to $61.7 \%$ in 2011 [14]. Minimally invasive surgery improved outcomes including decreased hospital stay, increased patient quality of life, consistency with patient preference, and enhanced cosmesis [15]. These factors have been considered as important drivers of cost and efficiency in the era of the Affordable Care Act [16].

Compared to other routes, TAH rates among patients with uterine cancer were higher in morbidly obese women compared to women with lower BMI levels. This might be explained by the increased and persistent technical challenges encountered by surgeons during minimally invasive surgery in patients with higher degrees of obesity [17]. Excessive adiposity poses several challenges to the surgical team, including poor patient tolerance to Trendelenburg positioning and positive intra-abdominal pressure, surgeons' fatigue, and the inability to correctly expose and develop the anatomical spaces [18].

On the other hand, TAH was found to be associated with an increased risk for perioperative complications when compared with other surgical routes. This is reflected by longer hospital stay, higher rates of surgical sites infections, and higher 30-day readmission rates. Since higher perioperative complications can compromise overall survival and success of adjuvant therapy, it is critical to take active measures to avoid 
TABLE 3: Perioperative outcomes among uterine cancer patients undergoing total laparoscopic hysterectomy, stratified by patient's body mass index, ACS-NSQIP, 2005-2013.

\begin{tabular}{|c|c|c|c|c|}
\hline \multirow[b]{2}{*}{ Characteristic/outcome } & \multicolumn{4}{|c|}{ BMI classification } \\
\hline & Ideal & Overweight & Obese & Morbidly obese \\
\hline Patient age (years) ${ }^{\mathrm{a}}$ & $63(55-72)$ & $65(57-72) *$ & $63(57-70)$ & $60(54-66) *$ \\
\hline Operation time $(\mathrm{min})^{\mathrm{a}}$ & $145(113-194)$ & $159(119-205) *$ & $158(120-206) *$ & $171(134-218) *$ \\
\hline Length of hospital stay (days) ${ }^{\mathrm{a}}$ & $1(1-1)$ & $1(1-1)$ & $1(1-1)$ & $1(1-1) *$ \\
\hline Bleeding transf usion ${ }^{\mathrm{b}}$ & $16(2.8)$ & $12(1.7)$ & $18(1.3) *$ & $14(1.4)$ \\
\hline DVT/thrombophlebitis & $0(0.0)$ & $4(0.6)$ & $6(0.4)$ & $7(0.7)$ \\
\hline Superficial surgical site infection ${ }^{c}$ & $3(0.5)$ & $4(0.6)$ & $13(1.0)$ & $13(1.3)$ \\
\hline Open wound/wound infection ${ }^{\mathrm{d}}$ & $1(0.2)$ & $1(0.1)$ & $7(0.5)$ & $13(1.3) *$ \\
\hline Deep incisional surgical site infection ${ }^{\mathrm{e}}$ & $0(0.0)$ & $1(0.1)$ & $2(0.1)$ & $5(0.5)$ \\
\hline Any infection listed above & $4(0.7)$ & $6(0.9)$ & $22(1.6)$ & $31(3.0) *$ \\
\hline Any adverse outcome listed above & $20(3.5)$ & $20(2.9)$ & $44(3.3)$ & $51(5.0)$ \\
\hline Readmission within 30 days $^{\mathrm{f}}$ & $15(2.8)$ & $23(3.6)$ & $50(3.9)$ & $41(4.2)$ \\
\hline
\end{tabular}

ACS NSQIP = American College of Surgeons National Surgical Quality Improvement Program; BMI = body mass index; DVT = deep vein thrombosis. * P-value $<0.05$ from either a Wilcoxon-Mann Whitney test (continuous variables) or chi-square test (categorical variables). For each outcome, three tests are performed: overweight vs. ideal BMI, obese vs. ideal BMI, morbidly obese vs. ideal BMI.

${ }^{\mathrm{a}}$ Values presented as median (Q1-Q3); all others are presented as frequency (\%).

${ }^{\mathrm{b}}$ At least 1 unit of packed or whole red blood cells given from the surgical start time up to and including 72 hours postoperatively.

${ }^{c}$ Infection that occurs within 30 days after the operation and the infection involves only skin or subcutaneous tissue of the incision.

${ }^{d}$ Preoperative evidence of a documented open wound at the time of the principal operative procedure. An open wound is a breach in the integrity of the skin or separation of skin edges and includes open surgical wounds, with or without cellulitis or purulent exudate. This does not include osteomyelitis or localized abscesses.

${ }^{\mathrm{e}}$ Infection that occurs within 30 days after the operation and the infection appears to be related to the operation and infection involved deep soft tissues (e.g., fascial and muscle layers) of the incision.

${ }^{\mathrm{f}}$ Readmission within 30 days was only available beginning in 2011; therefore, the percent provided reflects only the proportion of $2011-13$ cases who were readmitted.

or reduce the incidence of such complications [19]. With accumulating experience and increased training in minimally invasive surgeries, including robotically assisted procedures, the adoption of such techniques is likely to increase in the future; we suspect a concomitant improvement in perioperative outcomes in patients with uterine cancer [5].

In cases where pelvic lymph node dissection is performed, the preferred route for hysterectomy is either abdominal or laparoscopic. The utilization of TVH for uterine cancer surgery is controversial and the utility of nodal dissection in uterine cancer patients lacks a consensus opinion [20]. The role of TVH in uterine cancer depends on the type of the tumor, the stage of tumor, BMI, and presence of comorbidities. For stage I grade I uterine cancer, TVH may be reasonable, especially if CA- 125 level $<20 \mathrm{U} / \mathrm{mL}$ because of the low likelihood of extrauterine tumor invasion [21]. TVH utilization is limited in more advanced uterine cancer due to the limited ability to complete cancer staging.

In comparison with higher degrees of obesity, ideal body weight was found to be associated with the most favorable perioperative outcomes. This is supported by other studies showing obesity to be associated with increased complication rates in elective hysterectomy procedures, independent of the surgical route. Morbid obesity was found to be associated with increased conversion of laparoscopic surgery to laparotomy and less complete lymph node dissection [19, 22, 23]. Obesity is now considered as a pandemic with increasing prevalence [24]. It has been shown that patients who are obese experience some of the greatest differential benefits from minimally invasive techniques [10]. Obesity increased the risk of unintended conversion to laparotomy, where patients with BMI $>40$ have 4-fold increase in the conversion rate [18].

The ACS-NSQIP database that was used in this study represents a major strength due to its multi-institutional nature and is widely considered to be accurate, reproducible, and reliable. Data are collected by specially trained surgical clinical nurse reviewers who collect more than 100 clinical variables, including preoperative risk factors, intraoperative variables, and 30-day postoperative mortality and morbidity outcomes for patients undergoing major surgical procedures [25].

A weakness of this study is its observational nature. Although clinical trials can be the best research path to delineate optimum surgical approach for uterine cancer in morbidly obese patients, observational studies can be invaluable tool for hypothesis generation and prediction of patients who are at higher risk of complication of a certain therapeutic approach. Another weakness of the study is the fact that it lacked data on patient survival and its association with BMI categories and the inability to differentiate between conventional laparoscopic and robotic procedures. The data in the ACS-NSQIP are only from participating hospitals and, despite being distributed throughout USA, they do not collectively represent a statistically selected nationally representative sample. This study also lacks data regarding lymph node dissection; traditionally obesity is thought to be associated with less complete lymph node dissection; interestingly, in a study by Uccella et al, it was found that the 
number of lymph nodes removed was not affected by BMI [18]. TLH is currently the most commonly performed route for hysterectomy for patients with uterine cancer, regardless of the degree of obesity. Other confounding variables including surgical experience, hospital to hospital variation, and ethnicity could not be controlled for in this analysis.

Obesity poses an important challenge for the surgeon in selecting the surgical modality that balances between the technical difficultly and obtaining the best perioperative surgical outcomes.

\section{Abbreviations \\ BMI: $\quad$ Body mass index \\ ACS-NSQIP: American College of Surgeons-National Surgical Quality Improvement Program \\ CPT: $\quad$ Current procedural terminology \\ ICD-9-CM: International Classification of Disease, Ninth Revision, Clinical Modification \\ TAH: $\quad$ Total abdominal hysterectomy \\ TVH: Total vaginal hysterectomy \\ LAVH: $\quad$ Laparoscopic assisted vaginal hysterectomy \\ TLH: Total laparoscopic hysterectomy.}

\section{Data Availability}

Data are available upon request.

\section{Disclosure}

An earlier abstract of this paper was presented in the following link: http://www.jmig.org/article/S1553-4650(16)30281-3/ fulltext, November-December, 2016, Volume 23, Issue 7. A preliminary version of this data was presented at Minimally Invasive Surgery Week 2016-SLS Annual Meeting, September 2016, Boston, Massachusetts; the AAGL-Global Congress on Minimally Invasive Gynecology, November 2016, Orlando, FL; and ACOG Annual Meeting, May 2017, San Diego, California.

\section{Conflicts of Interest}

Drs. Entidhar Al Sawah, Jason L. Salemi, Mitchel Hoffman, Anthony N. Imudia, and Emad Mikhail report no conflicts of interest.

\section{Supplementary Materials}

Table S1: perioperative outcomes among uterine cancer patients with ideal BMI, stratified by route of hysterectomy, ACS-NSQIP, 2005-2013. Table S2: perioperative outcomes among uterine cancer patients with overweight BMI, stratified by route of hysterectomy, ACS-NSQIP, 2005-2013. Table S3: perioperative outcomes among uterine cancer patients with obese BMI, stratified by route of hysterectomy, ACS-NSQIP, 2005-2013. Table S4: perioperative outcomes among uterine cancer patients with morbidly obese BMI, stratified by route of hysterectomy, ACS-NSQIP, 2005-2013. (Supplementary Materials)

\section{References}

[1] J. Cardenas-Goicoechea, A. Shepherd, M. Momeni et al., "Survival analysis of robotic versus traditional laparoscopic surgical staging for endometrial cancer," American Journal of Obstetrics \& Gynecology, vol. 210, no. 2, pp. 160.el-160.e11, 2014.

[2] FF. Ferri, "Ferri's clinical advisor," in 5 books in 1, Ferri's clinical advisor 2014, 5 books in 1, 2014.

[3] G. H. Eltabbakh, M. I. Shamonki, J. M. Moody, and L. L. Garafano, "Hysterectomy for obese women with endometrial cancer: Laparoscopy or laparotomy?" Gynecologic Oncology, vol. 78, no. 3 I, pp. 329-335, 2000.

[4] M. Q. Bernardini, L. T. Gien, H. Tipping, J. Murphy, and B. P. Rosen, "Surgical outcome of robotic surgery in morbidly obese patient with endometrial cancer compared to laparotomy," International Journal of Gynecological Cancer, vol. 22, no. 1, pp. 76-81, 2012.

[5] T. N. Payne and M. C. Pitter, "Robotic-assisted surgery for the community gynecologist: Can it be adopted?" Clinical Obstetrics and Gynecology, vol. 54, no. 3, pp. 391-411, 2011.

[6] D. Sarlos and L. A. Kots, "Robotic versus laparoscopic hysterectomy: A review of recent comparative studies," Current Opinion in Obstetrics and Gynecology, vol. 23, no. 4, pp. 283-288, 2011.

[7] R. Ballard-Barbash and C. A. Swanson, "Body weight: estimation of risk for breast and endometrial cancers," American Journal of Clinical Nutrition, vol. 63, no. 3, pp. 437S-441S, 1996.

[8] D. Aune, D. A. Navarro Rosenblatt, D. S. M. Chan et al., "Anthropometric factors and endometrial cancer risk: A systematic review and dose-response meta-analysis of prospective studies," Annals of Oncology, vol. 26, no. 8, pp. 1635-1648, 2015.

[9] R. W. Holloway and S. Ahmad, "Robotic-assisted surgery in the management of endometrial cancer," Journal of Obstetrics and Gynaecology Research, vol. 38, no. 1, pp. 1-8, 2012.

[10] E. Mikhail, B. Miladinovic, V. Velanovich, M. A. Finan, S. Hart, and A. N. Imudia, "Association between obesity and the trends of routes of hysterectomy performed for benign indications," Obstetrics \& Gynecology, vol. 125, no. 4, pp. 912-918, 2015.

[11] S. F. Khuri, J. Daley, W. Henderson et al., "The Department of Veterans Affairs' NSQIP: The first national, validated, outcomebased, risk-adjusted, and peer-controlled program for the measurement and enhancement of the quality of surgical care," Annals of Surgery, vol. 228, no. 4, pp. 491-507, 1998.

[12] W. G. Henderson and J. Daley, "Design and statistical methodology of the National Surgical Quality Improvement Program: why is it what it is?" The American Journal of Surgery, vol. 198, no. 5, pp. S19-S27, 2009.

[13] E. von Elm, D. G. Altman, M. Egger et al., "The strengthening the reporting of observational studies in epidemiology (STROBE) statement: guidelines for reporting observational studies," PLoS Medicine, vol. 4, no. 10, article e297, 2007.

[14] J. D. Wright, W. M. Burke, A. I. Tergas et al., "Comparative effectiveness of minimally invasive hysterectomy for endometrial cancer," Journal of Clinical Oncology, vol. 34, no. 10, pp. 1087-1096, 2016.

[15] M. Frumovitz, P. T. Ramirez, M. Greer et al., "Laparoscopic training and practice in gynecologic oncology among Society of Gynecologic Oncologists members and fellows-in-training," Gynecologic Oncology, vol. 94, no. 3, pp. 746-753, 2004. 
[16] E. Litvak and M. Bisognano, "Analysis \& commentary: More patients, less payment: Increasing hospital efficiency in the aftermath of health reform," Health Affairs, vol. 30, no. 1, pp. 7680, 2011.

[17] G. S. Leiserowitz, G. Xing, A. Parikh-Patel et al., "Laparoscopic versus abdominal hysterectomy for endometrial cancer comparison of patient outcomes," International Journal of Gynecological Cancer, vol. 19, no. 8, pp. 1370-1376, 2009.

[18] S. Uccella, M. Bonzini, S. Palomba et al., "Impact of Obesity on Surgical Treatment for Endometrial Cancer: A Multicenter Study Comparing Laparoscopy vs Open Surgery, with Propensity-Matched Analysis," Journal of Minimally Invasive Gynecology, vol. 23, no. 1, pp. 53-61, 2016.

[19] F. Bouwman, A. Smits, A. Lopes et al., "The impact of BMI on surgical complications and outcomes in endometrial cancer surgery - An institutional study and systematic review of the literature," Gynecologic Oncology, vol. 139, no. 2, pp. 369-376, 2015.

[20] S. Uccella, K. C. Podratz, G. D. Aletti, and A. Mariani, "Lymphadenectomy in endometrial cancer," The Lancet, vol. 373, no. 9670, p. 1170, 2009.

[21] A. K. Sood, R. E. Buller, R. A. Burger, J. D. Dawson, J. I. Sorosky, and M. Berman, "Value of preoperative CA 125 level in the management of uterine cancer and prediction of clinical outcome," Obstetrics \& Gynecology, vol. 90, no. 3, pp. 441-447, 1997.

[22] K. Matsuo, C. E. Jung, M. S. Hom et al., "Predictive Factor of Conversion to Laparotomy in Minimally Invasive Surgical Staging for Endometrial Cancer," International Journal of Gynecological Cancer, vol. 26, no. 2, pp. 290-300, 2016.

[23] T.-J. Kim, G. Yoon, Y.-Y. Lee et al., "Robotic high para-aortic lymph node dissection with high port placement using same port for pelvic surgery in gynecologic cancer patients," Journal of Gynecologic Oncology, vol. 26, no. 3, pp. 222-226, 2015.

[24] M. Korenkov and S. Sauerland, "Clinical update: bariatric surgery," The Lancet, vol. 370, no. 9604, pp. 1988-1990, 2007.

[25] P. R. Fuchshuber, W. Greif, C. R. Tidwell et al., "The power of the National Surgical Quality Improvement Program-achieving a zero pneumonia rate in general surgery patients.," The Permanente Journal, vol. 16, no. 1, pp. 39-45, 2012. 


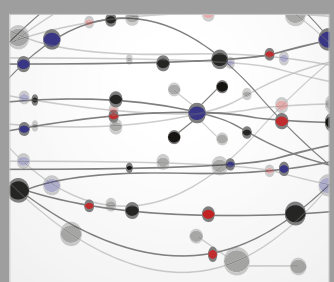

The Scientific World Journal
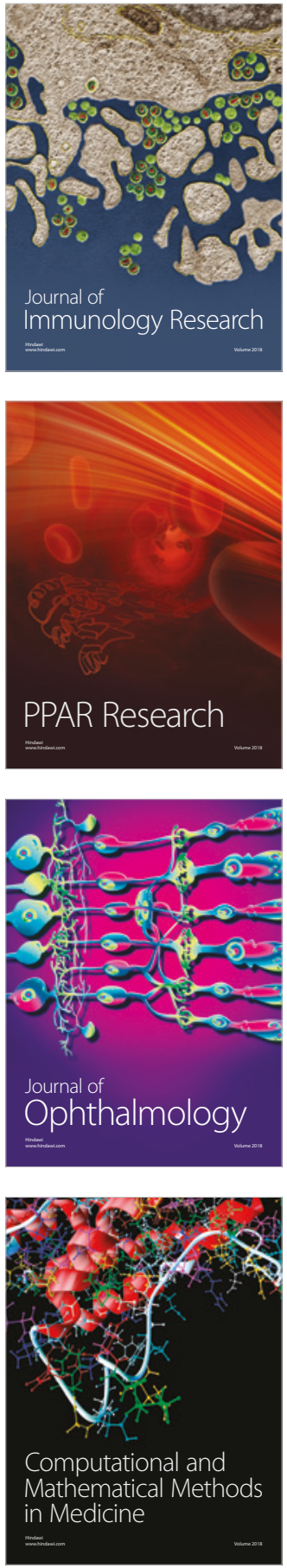

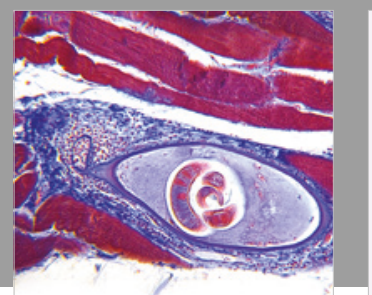

Gastroenterology Research and Practice

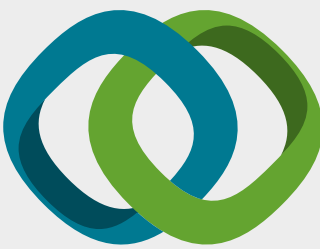

\section{Hindawi}

Submit your manuscripts at

www.hindawi.com
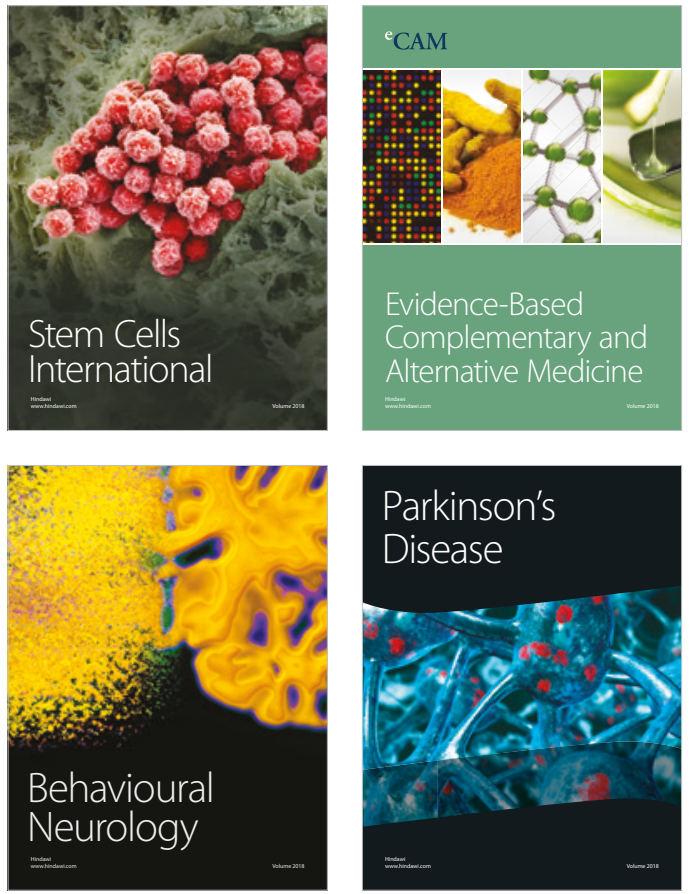

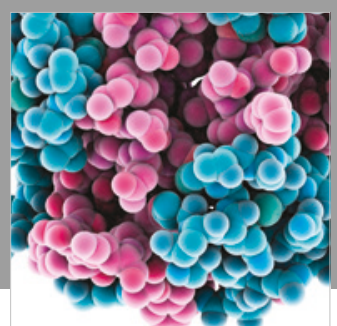

ournal of

Diabetes Research

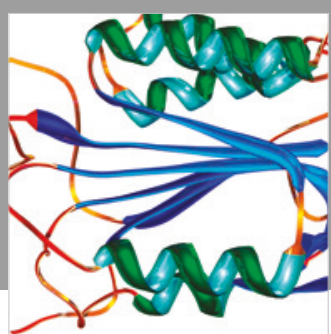

Disease Markers
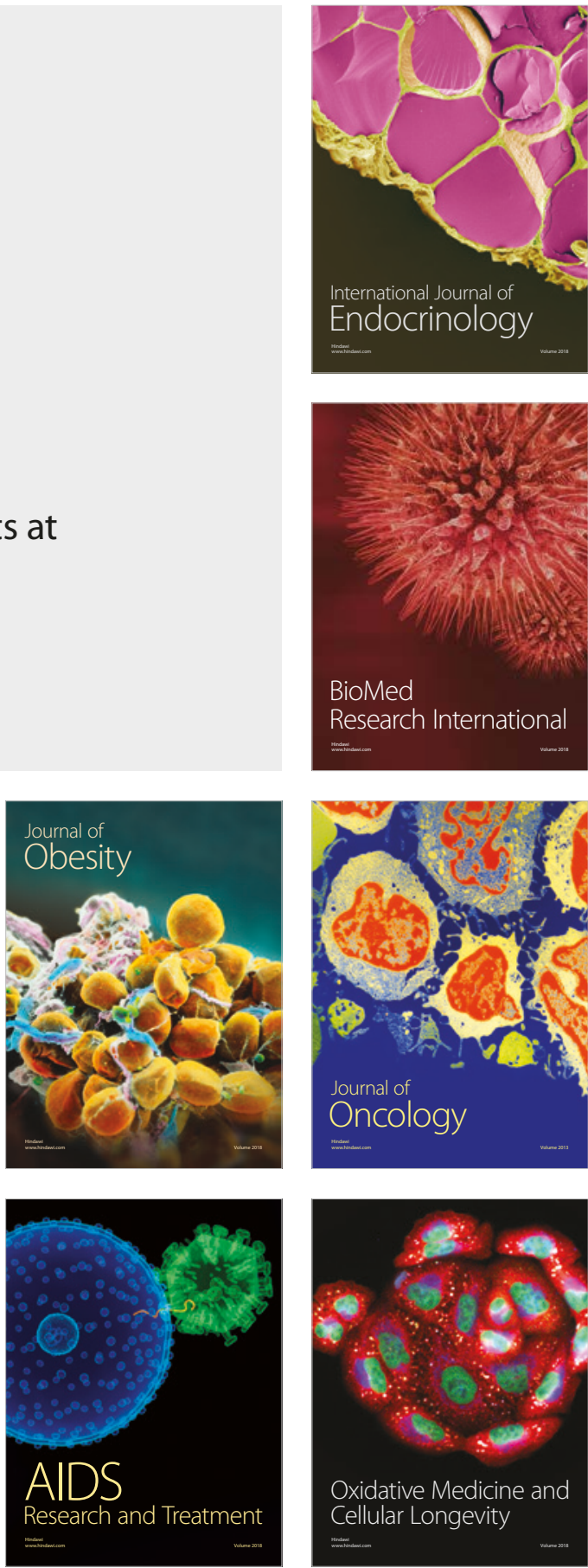